
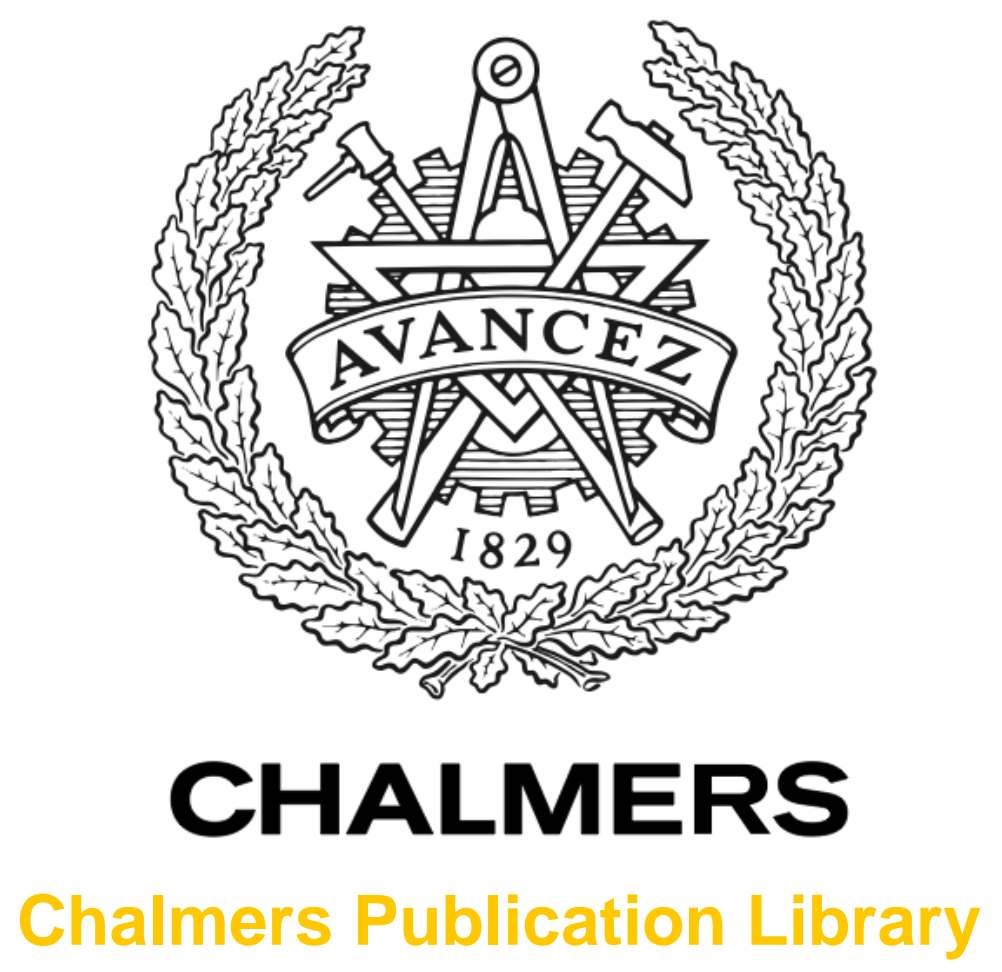

\title{
Approximate boundary conditions for a fluid-loaded elastic plate
}

This document has been downloaded from Chalmers Publication Library (CPL). It is the author's version of a work that was accepted for publication in:

Journal of the Acoustical Society of America (ISSN: 0001-4966)

Citation for the published paper:

Johansson, M. ; Folkow, P. ; Hägglund, A. et al. (2005) "Approximate boundary conditions for a fluid-loaded elastic plate". Journal of the Acoustical Society of America, vol. 118(6), pp. 3436-3446.

http://dx.doi.org/10.1121/1.2126927

Downloaded from: http://publications.lib.chalmers.se/publication/12172

Notice: Changes introduced as a result of publishing processes such as copy-editing and formatting may not be reflected in this document. For a definitive version of this work, please refer to the published source. Please note that access to the published version might require a subscription.

Chalmers Publication Library (CPL) offers the possibility of retrieving research publications produced at Chalmers University of Technology. It covers all types of publications: articles, dissertations, licentiate theses, masters theses, conference papers, reports etc. Since 2006 it is the official tool for Chalmers official publication statistics. To ensure that Chalmers research results are disseminated as widely as possible, an Open Access Policy has been adopted.

The CPL service is administrated and maintained by Chalmers Library. 


\section{Approximate boundary conditions for a fluid-loaded}

\section{elastic plate ${ }^{\mathrm{a}}$}

M. Johansson, P. D. Folkow ${ }^{\mathrm{b}}$, A. M. Hägglund, and P. Olsson

Department of Applied Mechanics

Chalmers University of Technology,

SE-41296 Göteborg, Sweden.

Running title: Approximate boundary conditions

\footnotetext{
a Submitted in October 2004 to the Journal of the Acoustical Society of America.

b Electronic mail: peter.folkow@me.chalmers.se
} 


\begin{abstract}
Approximate boundary conditions for an infinite elastic layer immersed in a fluid are derived. By using series expansions in the thickness coordinate of the plate fields, the displacements fields are eliminated adopting the three-dimensional equations of motion. The sums and differences of the boundary pressure fields and their normal derivatives are related through a set of approximate boundary conditions; one symmetric and one antisymmetric. These equations involve powers in the layer thickness together with partial derivatives with respect to time as well as the spatial variables in the plate plane. The approximate boundary conditions can be truncated to an arbitrary order, and explicit relations are presented including terms of order five. Comparisons are made with effective boundary conditions using classical plate theories. The numerical examples involve reflection and transmission of plane waves incident on the plate at different angles, as well as the pressure fields due to a line force. Three fluid loading cases are studied: modest, heavy and light loadings. The results using truncated approximate boundary conditions are compared to exact and classical plate solutions. The examples show that the accuracies of the power series approximations of order three and higher are very good in the frequency interval considered.
\end{abstract}

PACS numbers: 43.20.Gp, 43.20.Tb, 43.40.Rj. 


\section{INTRODUCTION}

It is a well-known fact that interfaces between bodies and surfaces of bodies exhibit properties that may be strikingly different from those of the bulk of their interiors. Indeed, surface physics as well as the physics of thin films are distinct branches of physics, studied in their own right. In applied mechanics, there are quite a number of situations where properties of thin interface layers must be analyzed. One example of such is scattering of waves from structural elements, where the thickness of the element in question is small in comparison with the wavelengths involved. Here, the elementary theories for membranes, shells and plates are often applicable. Besides these classical structural elements, there are other approximate theories that model various kinds of thin layers. As these theories may greatly simplify the analysis, thin layer theories have been studied extensively in the literature.

The effects from thin layers on the surroundings may be studied using approximate (effective) boundary and interface conditions. Such methods have been used in many areas, for instance acoustics [1], electromagnetics [2] and elastodynamics [1, 3, 4, 5]. Matrix formulations are adopted in Refs. 3 and 4, where the matrix elements are asymptotically expanded in the layer thickness. Elimination of the internal fields using series expansions together with boundary and interface conditions are used in Refs. 1 and 2 . Another series expansion approach is presented by Johansson and Niklasson [5], where methods for obtaining approximate boundary conditions to an (in principle) arbitrary order are presented. A somewhat different technique is adopted in the present paper, using systematic series 
expansions in the thickness coordinate. In spite of the seemingly different approaches used in the papers cited above, the various techniques are actually closely related.

In the work by Bövik [1], various combinations of surrounding media and interface materials are treated. The approach allows for the thin layer to have a fairly general curved shape in three dimensions, albeit with a constant thickness. The method used is a systematic elimination of the internal fields, and the layer is replaced by a single curved mathematical surface of discontinuity for the field variables in the surrounding media. The discontinuities are specified in such a manner that the influence of the layer is reproduced to within terms of second order in the thickness. However, the work by Bövik does not cover all combinations that may in practice occur. In fact, the case of an elastic layer immersed in a (non-viscous) fluid is not covered (while some apparently more complex cases are). The reason for these lacunae are stated by Bövik to be the fact that the rational elimination process that is used, breaks down when there are more degrees of freedom in the layer than in the surrounding medium.

The case of a planar thin elastic layer immersed in a fluid has been studied by many authors. In most works, the layer is modelled using the classical plate theories due to Kirchhoff [6] or Mindlin [7]. Various sorts of higher order plate theories may also be applicable in this context $[8,9,10,11]$. In the present paper the approximate boundary conditions are obtained by adopting a series expansion technique previously used in the derivation of higher order plate equations by Losin. $[12,13]$ The apparent restriction that hampered the elimination process used by Bövik is thus lifted, with a refinement of the method to include higher order terms. The present approach resembles the procedures 
employed in Ref. 5, which in turn is based on the higher order plate equation derivation presented by Boström et al.[14]. Hence, by generalizing the techniques adopted by Losin, $[12,13]$ the structural form of the approximate boundary conditions to an arbitrary order are obtained. Explicit expressions including terms up to order five in the thickness are presented and compared to corresponding expressions using classical plate theories. In order to illustrate the results using different theories, numerical examples are presented for three fluid loading cases. In the first examples, acoustic reflection from and transmission through an infinite elastic layer for plane waves are studied. This subject has been investigated by many authors. $[6,15,16,17,18]$ The next examples consider the pressure fields on the plate surfaces due to either symmetric or antisymmetric harmonic line forces. Sound radiation by plates excited locally by forces is an important problem of structural acoustics, extensively studied in the literature [6, 19, 20, 21, 22, 23].

\section{GOVERNING EQUATIONS}

Consider a plate of thickness $h$, bounded by the two planes $z= \pm h / 2$. The plate is surrounded by a linear non-viscous fluid with density $\rho_{f}$. The plate is homogeneous, isotropic and linearly elastic with density $\rho$ and Lamé constants $\lambda$ and $\mu$. The threedimensional equations of motion in the plate for the displacement components $u, v$ and $w$ 
are

$$
\begin{gathered}
(\lambda+\mu)\left(\partial_{x}^{2} u+\partial_{x y}^{2} v+\partial_{x z}^{2} w\right)+\mu\left(\partial_{x}^{2} u+\partial_{y}^{2} u+\partial_{z}^{2} u\right)=\rho \partial_{t}^{2} u \\
(\lambda+\mu)\left(\partial_{x y}^{2} u+\partial_{y}^{2} v+\partial_{y z}^{2} w\right)+\mu\left(\partial_{x}^{2} v+\partial_{y}^{2} v+\partial_{z}^{2} v\right)=\rho \partial_{t}^{2} v \\
(\lambda+\mu)\left(\partial_{x z}^{2} u+\partial_{y z}^{2} v+\partial_{z}^{2} w\right)+\mu\left(\partial_{x}^{2} w+\partial_{y}^{2} w+\partial_{z}^{2} w\right)=\rho \partial_{t}^{2} w .
\end{gathered}
$$

Partial derivatives are expressed in accordance with $\partial_{x}=\partial / \partial x$ etc.. Stress continuity at $z= \pm h / 2$ gives the boundary conditions (following Hooke's law)

$$
\begin{gathered}
\mu\left(\partial_{z} u+\partial_{x} w\right)=0 \\
\mu\left(\partial_{z} v+\partial_{y} w\right)=0 \\
\lambda\left(\partial_{x} u+\partial_{y} v\right)+(\lambda+2 \mu) \partial_{z} w=-p+F .
\end{gathered}
$$

The pressure from the surrounding fluid is denoted $p$, while $F$ is due to additive distributed forces at the boundaries acting outward in the normal direction. Vertical displacement continuity at $z= \pm h / 2$ together with the momentum equation for the fluid gives the additional boundary condition

$$
\partial_{t}^{2} w=-\partial_{z} p / \rho_{f}
$$

The main objective is to eliminate the plate fields $u, v$ and $w$ in the equations above so as to obtain approximate differential equations in terms of the fluid pressure $p$ and its normal derivative $\partial_{z} p$ at the boundaries. This is accomplished by first performing a power series expansion in the thickness coordinate $h$ of the left hand sides of the boundary conditions (4)-(7). These expansions are then combined with the equations of motion (1)-(3) to give the desired result. 
So, for the elimination process, it is convenient to proceed in terms of the differences and sums of the boundary conditions, as is done by Losin $[12,13]$. The boundary conditions (4)-(7) may thus be rewritten using $\Delta f=f(x, y, h / 2, t)-f(x, y,-h / 2, t)$ and $\Sigma f=$ $f(x, y, h / 2, t)+f(x, y,-h / 2, t)$, where $f$ is any of the boundary fields. For the plate fields, the sums and differences can be expanded in power series according to Maclaurin's formula

$$
\begin{aligned}
& \Delta f=2 \sum_{j=0}^{n-1} \frac{\partial_{z}^{2 j+1} f_{0}}{(2 j+1) !}\left(\frac{h}{2}\right)^{2 j+1}+\mathcal{O}\left(h^{2 n+1}\right), \\
& \Sigma f=2 \sum_{j=0}^{n-1} \frac{\partial_{z}^{2 j} f_{0}}{(2 j) !}\left(\frac{h}{2}\right)^{2 j}+\mathcal{O}\left(h^{2 n}\right),
\end{aligned}
$$

where $\partial_{z}^{m} f_{0}=\left.\left(\partial_{z}^{m} f(x, y, z, t)\right)\right|_{z=0}$.

The various plate fields present on the left hand sides of the boundary conditions may be expanded in such a way, which for Eq. (6) gives

$$
\begin{aligned}
& \Delta p-\Delta F=\sum_{j=0}^{n-1} \mathcal{A}_{1, j} \partial_{z}^{2 j+1} u_{0}+\mathcal{A}_{2, j} \partial_{z}^{2 j+1} v_{0}+\mathcal{A}_{3, j} \partial_{z}^{2 j+2} w_{0}+\mathcal{O}\left(h^{2 n+1}\right), \\
& \Sigma p-\Sigma F=\sum_{j=0}^{n-1} \mathcal{A}_{4, j} \partial_{z}^{2 j} u_{0}+\mathcal{A}_{5, j} \partial_{z}^{2 j} v_{0}+\mathcal{A}_{6, j} \partial_{z}^{2 j+1} w_{0}+\mathcal{O}\left(h^{2 n}\right)
\end{aligned}
$$

while Eq. (7) becomes

$$
\begin{aligned}
\Delta \partial_{z} p & =\sum_{j=0}^{n-1} \mathcal{A}_{7, j} \partial_{z}^{2 j+1} w_{0}+\mathcal{O}\left(h^{2 n+1}\right), \\
\Sigma \partial_{z} p & =\sum_{j=0}^{n-1} \mathcal{A}_{8, j} \partial_{z}^{2 j} w_{0}+\mathcal{O}\left(h^{2 n}\right) .
\end{aligned}
$$

The operators $\mathcal{A}_{i, j}$ involve powers of the plate thickness as well as possible time derivatives and spatial derivatives in the $(x y)$-plane. When studying the differential orders $\partial_{z}$ in Eqs. (10)-(13), it is seen that both $\Delta p$ and $\Sigma \partial_{z} p$ involve even orders acting on $w_{0}$ while $\Delta p$ 
involves odd orders acting on both $u_{0}$ and $v_{0}$. The opposite case holds for $\Sigma p$ and $\Delta \partial_{z} p$. It is therefore possible to obtain two separate relations between these fields; an antisymmetric case relating $\Delta p$ and $\Sigma \partial_{z} p$ and a symmetric case relating $\Sigma p$ and $\Delta \partial_{z} p$. Both cases may be treated similarly. For the antisymmetric case, Eqs. (10) and (13) consist of $3 n+3$ unknowns $\left(\Delta p, \Sigma \partial_{z} p, \partial_{z} u_{0}, \ldots, \partial_{z}^{2 n-1} u_{0}, \partial_{z} v_{0}, \ldots, \partial_{z}^{2 n-1} v_{0}, w_{0}, \ldots, \partial_{z}^{2 n} w_{0}\right)$, so it is clear that $3 n$ additional equations are needed in order to obtain a relation between $\Delta p$ and $\Sigma \partial_{z} p$. The boundary conditions (4) and (5) can be expanded using either Eq. (8) or Eq. (9). For the antisymmetric case, equation (9) is to be used, giving

$$
\begin{aligned}
& 0=\sum_{j=0}^{n-1} \mathcal{B}_{1, j} \partial_{z}^{2 j+1} u_{0}+\mathcal{B}_{2, j} \partial_{z}^{2 j} w_{0}+\mathcal{O}\left(h^{2 n}\right), \\
& 0=\sum_{j=0}^{n-1} \mathcal{B}_{1, j} \partial_{z}^{2 j+1} v_{0}+\mathcal{B}_{3, j} \partial_{z}^{2 j} w_{0}+\mathcal{O}\left(h^{2 n}\right),
\end{aligned}
$$

where the operators $\mathcal{B}_{i, j}$ involve powers of the plate thickness as well as spatial derivatives in the $(x y)$-plane. The last $3 n-2$ equations are obtained by using the differential equations (1)-(3) for the midplane fields $u_{0}, v_{0}$ and $w_{0}$. Both Eqs. (1) and (2) involve solely even differential orders $\partial_{z}$ on $u_{0}$ and $v_{0}$ and odd orders on $w_{0}$, while the opposite situation holds for (3). Differentiating Eqs. (1) and (2) an odd number of times with respect to $z$ renders $2 n-2$ more equations. The last $n$ equations are obtained by using Eq. (3) and its even derivatives with respect to $z$.

Hence, it is possible to solve the two separate linear systems for the antisymmetric and 
symmetric cases, respectively, giving

$$
\begin{aligned}
& \left(\sum_{j=0}^{n-1} h^{2 j} \partial_{t}^{2} \mathcal{C}_{1, j}\right)(\Delta p-\Delta F)=\left(\sum_{j=1}^{n} h^{2 j-1} \mathcal{C}_{2, j}\right) \Sigma \partial_{z} p+\mathcal{O}\left(h^{2 n}\right) \\
& \left(\sum_{j=1}^{n} h^{2 j} \partial_{t}^{2} \mathcal{C}_{3, j}\right)(\Sigma p-\Sigma F)=\left(\sum_{j=1}^{n} h^{2 j-1} \mathcal{C}_{4, j}\right) \Delta \partial_{z} p+\mathcal{O}\left(h^{2 n}\right)
\end{aligned}
$$

The differential operators $\mathcal{C}_{i, j}$ involve $\nabla_{s}^{2 p} \partial_{t}^{2 j-2 p}$ for $p=0, \ldots, j$ with $\nabla_{s}^{2}=\partial_{x}^{2}+\partial_{y}^{2}$. Thus, their highest order purely spatial derivatives are $\nabla_{s}^{2 j}$, and their highest order purely time derivatives are $\partial_{t}^{2 j}$.

As the series expansion may be derived to any order, the accuracy of the solution can be made as good as one wishes [28]. The more terms involved in the series, the better approximation for shorter waves. However, the main interest in the results is perhaps to study expansions using a few terms, which corresponds to long wavelength and low frequency approximations.

Presenting the explicit relations for the antisymmetric case, Eq. (16) gives up to order $h^{5}$

$$
\begin{aligned}
& \frac{\rho_{f}}{\rho} \frac{\partial_{t}^{2}}{c_{s}^{2}}\left\{1+\frac{1}{2}\left(\frac{h}{2}\right)^{2}\left[-2 \nabla_{s}^{2}+(1+\gamma) \frac{\partial_{t}^{2}}{c_{s}^{2}}\right]\right. \\
& \left.+\frac{1}{24}\left(\frac{h}{2}\right)^{4}\left[8 \nabla_{s}^{4}-8(1+\gamma) \nabla_{s}^{2} \frac{\partial_{t}^{2}}{c_{s}^{2}}+\left(1+6 \gamma+\gamma^{2}\right) \frac{\partial_{t}^{4}}{c_{s}^{4}}\right]\right\}(\Delta p-\Delta F) \\
& =\left\{\frac{h}{2} \frac{\partial_{t}^{2}}{c_{s}^{2}}+\frac{1}{6}\left(\frac{h}{2}\right)^{3}\left[8(1-\gamma) \nabla_{s}^{4}-4(3-2 \gamma) \nabla_{s}^{2} \frac{\partial_{t}^{2}}{c_{s}^{2}}+(3+\gamma) \frac{\partial_{t}^{4}}{c_{s}^{4}}\right]\right. \\
& +\frac{1}{120}\left(\frac{h}{2}\right)^{5}\left[-32(1-\gamma) \nabla_{s}^{6}+16\left(4-2 \gamma-\gamma^{2}\right) \nabla_{s}^{4} \frac{\partial_{t}^{2}}{c_{s}^{2}}\right. \\
& \left.\left.-4\left(9+3 \gamma-4 \gamma^{2}\right) \nabla_{s}^{2} \frac{\partial_{t}^{4}}{c_{s}^{4}}+\left(5+10 \gamma+\gamma^{2}\right) \frac{\partial_{t}^{6}}{c_{s}^{6}}\right]\right\} \Sigma \partial_{z} p .
\end{aligned}
$$


In the symmetric case, Eq. (17) gives up to order $h^{5}$

$$
\begin{aligned}
& \frac{\rho_{f}}{\rho} \frac{\partial_{t}^{2}}{c_{s}^{2}}\left(-\nabla_{s}^{2}+\gamma \frac{\partial_{t}^{2}}{c_{s}^{2}}\right)\left\{\frac{h}{2}+\frac{1}{6}\left(\frac{h}{2}\right)^{3}\left[-2 \nabla_{s}^{2}+(1+\gamma) \frac{\partial_{t}^{2}}{c_{s}^{2}}\right]\right. \\
& \left.+\frac{1}{360}\left(\frac{h}{2}\right)^{5}\left[4 \nabla_{s}^{2}-(1+3 \gamma) \frac{\partial_{t}^{2}}{c_{s}^{2}}\right]\left[4 \nabla_{s}^{2}-(3+\gamma) \frac{\partial_{t}^{2}}{c_{s}^{2}}\right]\right\}(\Sigma p-\Sigma F) \\
& =\left\{\left[-4(1-\gamma) \nabla_{s}^{2}+\frac{\partial_{t}^{2}}{c_{s}^{2}}\right]+\frac{1}{6}\left(\frac{h}{2}\right)^{2}\left[8(1-\gamma) \nabla_{s}^{4}-4\left(2-\gamma^{2}\right) \nabla_{s}^{2} \frac{\partial_{t}^{2}}{c_{s}^{2}}+(1+3 \gamma) \frac{\partial_{t}^{4}}{c_{s}^{4}}\right]\right. \\
& +\frac{1}{120}\left(\frac{h}{2}\right)^{4}\left[-32(1-\gamma) \nabla_{s}^{6}+16\left(3-2 \gamma^{2}\right) \nabla_{s}^{4} \frac{\partial_{t}^{2}}{c_{s}^{2}}\right. \\
& \left.\left.-4\left(4+10 \gamma-5 \gamma^{2}-\gamma^{3}\right) \nabla_{s}^{2} \frac{\partial_{t}^{4}}{c_{s}^{4}}+\left(1+10 \gamma+5 \gamma^{2}\right) \frac{\partial_{t}^{6}}{c_{s}^{6}}\right]\right\} \Delta \partial_{z} p .
\end{aligned}
$$

In these equations, $c_{s}=\sqrt{\mu / \rho}$ is the transverse wave speed and $\gamma=\mu /(\lambda+2 \mu)$ is the squared quotient between the transverse and the longitudinal wave speeds. Note the factorized differential operator on the left hand side of Eq. (19). One consequence of this operator is that when $\left(-\nabla_{s}^{2}+\gamma \frac{\partial_{t}^{2}}{c_{s}^{2}}\right)(\Sigma p-\Sigma F)=0$, the normal motion of the plate boundaries is purely antisymmetric as $\Delta \partial_{z} p=0$. Speaking in terms of plane time-harmonic waves, this occurs when the horizontal phase speed in the fluid is equal to the longitudinal wave speed in the elastic layer. The same result is obtained using exact theory, so the factorization of the operator probably holds to arbitrary order.

Asymptotic expansions for plates without surrounding fluids have been studied by Losin $[12,13]$ and Boström et al.[14] In the works by Losin, where no boundary forces are included, the right hand sides of Eqs. (18) and (19) can be identified. Boström et al. investigated the antisymmetric case including boundary forces, and the result may be shown to be in line with Eq. (18).

It is instructive to compare these asymptotic equations with the results from classical 
plate theory. For the antisymmetric case, the most simple approximation is to study the motion of a perfectly limp layer, giving

$$
\frac{\rho_{f}}{\rho}(\Delta p-\Delta F)=\frac{h}{2} \Sigma \partial_{z} p
$$

This result is found in Eq. (18) when terms up to order $h$ are included. A more refined theory that includes plate bending is the classic Kirchhoff equation, which in this context is written

$$
\frac{\rho_{f}}{\rho} \frac{\partial_{t}^{2}}{c_{s}^{2}}(\Delta p-\Delta F)=\left\{\frac{h}{2} \frac{\partial_{t}^{2}}{c_{s}^{2}}+\frac{1}{6}\left(\frac{h}{2}\right)^{3}\left[8(1-\gamma) \nabla_{s}^{4}\right]\right\} \Sigma \partial_{z} p
$$

Hence, an extra term involving $h^{3}$ is added when compared to Eq. (20) due to the influence of the bending stiffness. It is clear from Eq. (18) that the Kirchhoff equation does not include the terms of order $h^{2}$ on the left hand side and only the first term of the $h^{3}$-terms on the right hand side. Turning into the Mindlin theory which includes both shear effects and rotary inertia, the equation becomes

$$
\begin{aligned}
& \frac{\rho_{f}}{\rho} \frac{\partial_{t}^{2}}{c_{s}^{2}}\left\{1+\frac{1}{2}\left(\frac{h}{2}\right)^{2}\left[-\frac{8}{3 \kappa}(1-\gamma) \nabla_{s}^{2}+\frac{2}{3 \kappa} \frac{\partial_{t}^{2}}{c_{s}^{2}}\right]\right\}(\Delta p-\Delta F) \\
& =\left\{\frac{h}{2} \frac{\partial_{t}^{2}}{c_{s}^{2}}+\frac{1}{6}\left(\frac{h}{2}\right)^{3}\left[8(1-\gamma) \nabla_{s}^{4}-\left(2+\frac{8}{\kappa}(1-\gamma)\right) \nabla_{s}^{2} \frac{\partial_{t}^{2}}{c_{s}^{2}}+\frac{2}{\kappa} \frac{\partial_{t}^{4}}{c_{s}^{4}}\right]\right\} \Sigma \partial_{z} p .
\end{aligned}
$$

This equation involves various terms of order $h^{2}$ and $h^{3}$ of which the coefficients differ from the corresponding terms presented in Eq. (18). Here $\kappa$ is the much debated shear coefficient which is an adjustment coefficient not appearing in Eq. (18). In the numerical examples presented below, $\kappa$ is chosen as $\kappa=5 /(6-\nu)$ in accordance with Ref. 14, where $\nu$ is the Poisson ratio.

In the symmetric case, the most simple approximation is to consider a layer in gener- 
alized plane stress giving

$$
\frac{\rho_{f}}{\rho} \frac{\partial_{t}^{2}}{c_{s}^{2}}\left\{\frac{h}{2}\left[-\nabla_{s}^{2}+\gamma \frac{\partial_{t}^{2}}{c_{s}^{2}}\right]\right\}(\Sigma p-\Sigma F)=\left[-4(1-\gamma) \nabla_{s}^{2}+\frac{\partial_{t}^{2}}{c_{s}^{2}}\right] \Delta \partial_{z} p
$$

which is just Eq. (19) up to order $h$. A higher order plate theory is due to Kane and Mindlin [24] which in this case becomes

$$
\begin{aligned}
& \frac{\rho_{f}}{\rho} \frac{\partial_{t}^{2}}{c_{s}^{2}}\left\{\frac{h}{2}\left[-\nabla_{s}^{2}+\gamma \frac{\partial_{t}^{2}}{c_{s}^{2}}\right]\right\}(\Sigma p-\Sigma F) \\
& =\left\{\left[-4 \kappa^{2}(1-\gamma) \nabla_{s}^{2}+\kappa^{2} \frac{\partial_{t}^{2}}{c_{s}^{2}}\right]+\frac{1}{6}\left(\frac{h}{2}\right)^{2}\left[2 \nabla_{s}^{4}-2(1+\gamma) \nabla_{s}^{2} \frac{\partial_{t}^{2}}{c_{s}^{2}}+2 \gamma \frac{\partial_{t}^{4}}{c_{s}^{4}}\right]\right\} \Delta \partial_{z} p .
\end{aligned}
$$

This equation involves terms up to order $h^{2}$, but the coefficients of the differential operators on the right hand side are different from the corresponding terms given in Eq. (19).

\section{NUMERICAL CALCULATIONS OF TRANSMIS- SION AND REFLECTION}

To validate the various approximate equations presented above, the reflection from and transmission through the plate may be considered for $F=0$. The wave propagation in the fluid medium is here assumed to follow the simple wave equation

$$
\nabla^{2} p=\partial_{t}^{2} p / c_{f}^{2}
$$

where $c_{f}$ is the wave speed and $\nabla^{2}=\partial_{x}^{2}+\partial_{y}^{2}+\partial_{z}^{2}$. Consider $z<-h / 2$ and let plane waves propagate in the $(x z)$-plane towards $z=-h / 2$, giving rise to reflected and transmitted 
plane waves. Thus, the incident, reflected and transmitted waves may be expressed as

$$
\begin{array}{ll}
p_{i}=\mathrm{e}^{\mathrm{i}\left(k_{f}\left(\zeta x+\sqrt{1-\zeta^{2}} z\right)-\omega t\right)} & z<-h / 2, \\
p_{r}=R \mathrm{e}^{\mathrm{i}\left(k_{f}\left(\zeta x-\sqrt{1-\zeta^{2}} z\right)-\omega t\right)} & z<-h / 2, \\
p_{t}=T \mathrm{e}^{\mathrm{i}\left(k_{f}\left(\zeta x+\sqrt{1-\zeta^{2}} z\right)-\omega t\right)} & z>h / 2,
\end{array}
$$

where $k_{f}=\omega / c_{f}$ and $\zeta=\sin \phi ; \phi$ being the angle measured from the normal to the plate boundaries.

It is now straightforward to solve for $R$ and $T$ using either the exact three-dimensional equations of motion (1)-(3) with pertinent boundary conditions, or the asymptotic equations (18) and (19). As the loading is such that purely antisymmetric or symmetric modes will not be generated in the general case, the equations (18) and (19) are solved as a system. Only odd order expansions in the plate thickness are here of interest. The reason for this is that the quotient between the differences and the sums of the pressure fields $p$ and $\partial_{z} p$ are seen to be of order $h$. Hence, in Eqs. (18) and (19), every thickness order term on the left hand side is to be paired to a corresponding term on the right hand side for a correct truncation. The numerical results involve solutions based on the asymptotic $h, h^{3}$ and $h^{5}$ expansions, respectively. The range of applicability for each truncation level is hereby clearly visible. When performing comparisons to classical plate theories, mainly solutions involving Kirchhoff theory (21) and Mindlin theory (22) are presented. These equations have been used extensively in the literature and are suitable for comparisons since the antisymmetric motions are prevailing in most of the examples presented below. When the influence from the symmetric motions is pronounced, numerical results are explicitly given 
using classical theories covering both antisymmetric and symmetric motions. Note that in all the various plate equations that are studied, $R^{2}+T^{2}=1$ as no losses are incorporated in the models.

It is convenient to introduce various non-dimensional parameters, besides $\gamma$, that appear in the equations:

$$
\alpha=\rho_{f} / \rho, \quad \beta=c_{f}^{2} / c_{s}^{2}, \quad \Omega=k_{s} h,
$$

where $k_{s}=\omega / c_{s}$.

\section{III.1 Aluminum-Water}

Consider a case of modest loading, where an aluminum plate is surrounded by water. Here, the various non-dimensional parameters become $\alpha \approx 0.37, \beta \approx 0.23$ and $\gamma \approx 0.25$.

Using exact theory, the transmission coefficient modulus is displayed in a 3D-plot Fig. 1(a). The figure shows the dependence of $|T|$ upon the frequency $\Omega$ and the angle of incidence, displayed through $\zeta$. These results are to be compared to the asymptotic $h, h^{3}$ and $h^{5}$ expansions as well as the classical Kirchhoff and Mindlin theories. Fig. 1(b) shows the modulus of $T$ when the angle of incidence is $\phi=45^{\circ}$. The result using the $h^{5}$ expansion is almost indistinguishable from the exact result over a large frequency interval. Mindlin and the $h^{3}$ results are of the same accuracy even though the former is somewhat better for lower frequencies, while the Kirchhoff theory is clearly inferior. The simple $h$ expansion is valid for low frequencies only. It completely misses the total transmission effect due to the flexural motion (closely related to the zeroth antisymmetric Lamb mode for a free plate) 
as expected. Note that the total transmission for the Kirchhoff and Mindlin theories occur when the horizontal wave number in the fluid, $k_{f} \zeta$, is equal to the plate wavenumber for a free plate. This behavior does not appear for the exact and asymptotic theories in the general case.

The dependence of $|T|$ upon the angle of incidence is presented in Fig. 1(c) for the frequency $\Omega=0.5$. The accuracy of the different approximations show a similar behavior as in Fig. 1(b). Note the rapid transition of the transmission coefficient from zero to unity around $\zeta \approx 0.28$, that is $\phi \approx 16^{\circ}$. This is associated with the first compressional mode (closely related to the zeroth symmetric Lamb mode for a free plate). The phenomenon is not captured by the Kirchhoff and Mindlin theories since they do not take symmetric motion into account. Another minor incorrectness with the Kirchhoff and Mindlin equations is that $|T|=1$ for the grazing angle, $\zeta=1$. The exact and asymptotic equations predict that the transmission modulus falls quickly to zero as $\zeta$ approaches unity. This behavior is also illustrated in Fig. 1(d) where the curves of total transmission are displayed. The curve related to the loci of the lowest flexural mode show once again that the Kirchhoff theory is inferior to the other higher order theories. Hence, the Kirchhoff equation is here not that well suited when predicting total transmission. The curve related to the lowest compressional mode shows very little frequency dependency. Here, the various asymptotic curves are indistinguishable from the exact curve. Moreover, there exists a curve just below $\zeta=1$ due to the $h$ expansion.

In order to perceive the range of accuracy for the different theories, the contour lines for one percent relative error in the transmission modulus are displayed in Fig. 2. The 
different theories involving terms of order $h^{3}$, that is Kirchhoff, Mindlin and asymptotic $h^{3}$ expansions, are presented in Fig. 2(a). The latter theory is seen to be within the error limit over a larger frequency interval, especially for $\phi<30^{\circ}$. In Fig. 2(b) similar contours for the asymptotic $h, h^{3}$ and $h^{5}$ theories are depicted, showing the range improvement using higher order theories. Considering an explicit example using a two mm thick aluminum plate immersed in water, the $h$ theory applies within the error limit for frequencies up to around $25 \mathrm{kHz}$, while the $h^{3}$ theory is valid up to $0.1 \mathrm{MHz}$ and $h^{5}$ theory up to $0.17 \mathrm{MHz}$.

In all the examples presented above, the comparisons are made with the purely antisymmetric classical plate equations due to Kirchhoff or Mindlin. As the asymptotic equations involve both symmetric and antisymmetric motions, the flexural plate equations can be combined with the classical symmetrical plate equations as well. Two different combinations are considered here; the Kirchhoff equation (21) together with the generalized plane stress equation (23), (K-GPS), as well as the Mindlin equation (22) together with the equation due to Kane and Mindlin (24), (M-KM). The main reason for choosing the M-KM solution is that it is based on refined antisymmetric and symmetric theories when compared to K-GPS; thus resembling the asymptotic $h^{3}$ to a greater extent. Several authors have studied the effects of various such combined equations. $[15,17,25,26]$ The equations derived by Lyamshev[25] are, after corrections, in line with the K-GPS relations. Freedman[15] adopted the generalized plane stress equation together with the Mindlin equation. However, some of his results exhibit some peculiar behavior not appearing when implemented here. The influence of the symmetric parts in both the K-GPS and the M-KM theories are best appreciated when compared to the results displayed in Fig. 1(c). First, both the com- 
bined classical theories feature the rapid transition of the transmission coefficient around $\zeta \approx 0.28$ due to the compressional mode, as expected. Second, the transmission modulus tends abruptly to zero as $\zeta$ approaches unity in both cases. Otherwise, there are small discrepancies between the combined theories (K-GPS and M-KM) and the corresponding antisymmetric theories (Kirchhoff and Mindlin) in this case.

\section{III.2 Polyethylene-Water}

In this section a light solid is immersed in water. The solid parameters are those of polyethylene, which represents a typical plastic material. The amplitudes are assumed to be low, so that elastic theory is applicable. The system is an example of heavy loading, where the non-dimensional parameters become $\alpha \approx 1.03, \beta \approx 0.71$ and $\gamma \approx 0.25$.

The exact transmission coefficient modulus is displayed in Fig. 3(a). It is seen that the transmission through the plate is pronounced for a large interval of both the frequency and the angle of incidence, compared to the aluminum-water case Fig. 1(a). This is due to the similarities in the plate and fluid impedances in this heavy loading example. When displaying the frequency dependence of the transmission coefficient modulus for the different approximate theories, it is seen that for most of the angles of incidence the classical plate equations reproduce the behavior less correctly than the asymptotic expansions, including the simple $h$ expansion. This indicates that the symmetric motion is of importance in this frequency interval. In the case of small angles of incidence, the classical theories are rather good as is shown in Fig. 3(b) when $\phi=15^{\circ}$. The plot displays the discrepancy of the 
asymptotic expansions as the frequency increases. Note the total reflection according to the $h^{3}$ theory, which is at a lower frequency than according to exact theory; $\Omega \approx 1.76$. The accuracies of the classical and asymptotic theories are clearly visible in Fig. 3(c) where the angle of incidence is varied for $\Omega=1$. The classical theories fail to illustrate the behavior of the transmission coefficient for most $\zeta$. There exists a rapid transition of $|T|$ from zero to unity around $\zeta \approx 0.5\left(\phi \approx 30^{\circ}\right)$ which corresponds to the lowest compressional mode. This behavior was also present in the aluminum-water case Fig. 1(c), where the relation to the zeroth symmetric Lamb mode was stated. However, total transmission occurs at $\zeta \approx 0.8$, which is not that close to the corresponding zeroth order antisymmetric Lamb mode for a free plate. In this case, neither the symmetric nor the antisymmetric part of the motion is prevailing [16]. This is easily seen by analyzing the equations for the asymptotic $h$ expansion, which solution is surprisingly accurate. Similar results are also clearly visible in Fig. 3(d), where the Kirchhoff and Mindlin plate theories obviously differ from the rest. Hence, comparisons to the free plate Lamb modes are of less importance in this heavy loading example, as expected [18].

As in the aluminum-water case, contour lines are displayed in Fig. 4. The results using Kirchhoff, Mindlin and asymptotic $h^{3}$ expansions are presented in Fig. 4(a), showing the superiority of the latter theory. Here, the lines corresponding to the classical theories are mostly on top of each other. The various asymptotic expansions are displayed in Fig. 4(b). The accuracies are quite good for all the expansions, beside around $\zeta=0.5$ where the relative errors become pronounced due to the transition from $T=0$.

Due to the importance of the symmetric motion in this heavy loading case, it is in- 
teresting to study the combined classical theories according to K-GPS and M-KM. When compared to the results presented in Fig. 3(c), both K-GPS and M-KM are considerably better than the classical antisymmetrical plate theories. The accuracies are displayed in Fig. 5(a), where the absolute values of the error in the transmission coefficient modulus, $|\Delta| T||$, are plotted. As in the aluminum-water case, the combined classical theories render more or less the correct behavior around the transition point $\zeta \approx 0.5$ as well as near $\zeta=1$. The contour lines for the three theories of order $h^{3}$, that is K-GPS, M-KM and asymptotic $h^{3}$, are given in Fig. 5(b). The improvement using the combined classical theories are clearly visible when compared to Fig. 4(a). However, Fig. 5 show that the the asymptotic $h^{3}$ and $h^{5}$ expansions are, in most cases, the best approximations.

\section{III.3 Steel-Air}

The last example considers a case of light fluid loading, a steel plate in air. The nondimensional parameters are $\alpha \approx 1.66 \times 10^{-4}, \beta \approx 0.011$ and $\gamma \approx 0.33$.

The pronounced impedance mismatch causes the plate to act as a sound barrier $(|T| \approx$ 0 ) for most combinations of $\Omega$ and $\zeta$. The transmission coefficient tends to unity for those $(\Omega, \zeta)$ which are close to the solutions of the dispersion relation for a free plate. In Fig. 6(a) the exact value of $\log |T|$ is plotted for some different angles of incidence, resembling the results presented in the aluminum-water case Fig. 1(a). The behavior of the transmission coefficient using the different plate equations is displayed in Fig. 6(b) for $\phi=10^{\circ}$. As in the aluminum-water case, the $h^{5}$ expansion result is very close to the exact one, while 
the $h$ expansion does not include the flexural mode. Fig. 6(c) shows the very sharp transition of the transmission coefficient from zero to unity for the compressional mode around $\phi=3.6^{\circ}$, which is not included in the Kirchhoff and Mindlin theories. The curves for total transmission are presented in Fig. 6(d) similar to Fig. 1(d).

\section{NUMERICAL CALCULATIONS FOR A LINE LOADING}

To further evaluate the asymptotic equations, the pressure fields on the plate boundaries due to applied forces are calculated. As the fluid-plate responses from time harmonic line forces have been studied by many authors [19, 20, 21, 22, 23], such problems are addressed here. The line forces are modelled according to $\Delta F=F_{a} \delta(x) \mathrm{e}^{-\mathrm{i} \omega t}$ in the antisymmetric case and $\Sigma F=F_{s} \delta(x) \mathrm{e}^{-\mathrm{i} \omega t}$ in the symmetric case. Each case is studied separately. As the problems become $y$ independent, this coordinate is suppressed throughout. The problems are solved by applying the Fourier transform in the $x$-coordinate. If the fluid is assumed to follow the wave equation (25), the transformed pressure fields are given by

$$
\begin{array}{ll}
\widehat{p}^{+}=p^{+} \mathrm{e}^{\mathrm{i}(k z-\omega t)}, & z>h / 2, \\
\widehat{p}^{-}=p^{-} \mathrm{e}^{\mathrm{i}(-k z-\omega t)}, & z<-h / 2,
\end{array}
$$

where $k=\sqrt{k_{f}^{2}-q^{2}} ; q$ being the transform variable. In the antisymmetric case $p^{-}=-p^{+}$ while in the symmetric case $p^{-}=p^{+}$. Thus, it is clear that Eqs. (27) and (28) can be 
combined to give

$$
\begin{array}{ll}
\Delta \widehat{p}(q)=\widehat{p}_{a}(q), & \Sigma \partial_{z} \widehat{p}(q)=\mathrm{i} k(q) \widehat{p}_{a}(q), \\
\Sigma \widehat{p}(q)=\widehat{p}_{s}(q), & \Delta \partial_{z} \widehat{p}(q)=\mathrm{i} k(q) \widehat{p}_{s}(q) .
\end{array}
$$

By using the relations in (29) and (30), it is straightforward to relate $p_{a}$ to $F_{a}$ as well as $p_{s}$ to $F_{s}$ in the approximate boundary conditions $(18)-(24)$. The transformed pressure fields at the boundaries can in each such case be expressed on the form

$$
\widehat{p}_{a, s}(q)=\frac{N_{a, s}(q)}{N_{a, s}(q)+k(q) D_{a, s}(q)} F_{a, s} .
$$

The various functions $N_{a, s}$ and $D_{a, s}$ involve polynomials in $q^{2}$. The numerators are recognized as the dispersion equations for fluid loaded plates, while the functions $D_{a, s}$ appear when studying the dispersion relations for the corresponding free plates. For the exact theory, separate antisymmetric and symmetric modes are easily obtained [27]. The pressure relations may still be written on the form of Eq. (31). Here $N_{a, s}$ and $D_{a, s}$ are even functions in $q$, including trigonometric functions. The functions $D_{a, s}$ are then recognized as the Rayleigh-Lamb frequency equations. It is instructive to note that the numerator and denominator of Eq. (31) in the exact cases may be expanded in the layer thickness coordinate $h$, giving the same quotients as for the asymptotic plate equations discussed above, see further Losin [28].

By using the inversion formula, the pressure fields $p_{a, s}(x)$ are obtained from $\widehat{p}_{a, s}(q)$ in Eq. (31) for each plate theory. The integrals are rewritten as cosine transforms, since the integrands are even in $q$. Analytical solution of these integrals are complicated to evaluate, 
even for the lowest order case. Therefore, they are computed numerically. The contour of integration is deformed into the fourth quadrant due to the poles and branch points on the real axis.

The numerical results Figs. 7-8 show the plate boundary pressure as a function of the distance from the line force. Only the pressure component in phase with the line force is presented. All three fluid loading situations are studied for both the antisymmetric and the symmetric cases. In order to easily distinguish between the results using various plate theories, the chosen frequency is quite high, $k_{s} h=1$. In Fig. 7, the antisymmetric motions for the different theories are displayed. The lowest order $h$ theory is not included here, as this simple approximation completely fails to predict the plate and fluid behavior in this loading case. From the figures, it is clear that the Kirchhoff theory is less accurate than the other more refined theories at this frequency level, as expected. The accuracies using the Mindlin equation and the asymptotic $h^{3}$ equation are of the same order. The results using the $h^{5}$ theory are very close to the exact curves. The corresponding symmetric cases are plotted in Fig. 8. Here, the curves using the approximate equations comprise the classic Kane-Mindlin equation as well as the three different asymptotic equations. It is seen from these results that the relations between the plate and fluid parameters influence the wave lengths in a more pronounced way than in the antisymmetric cases. In the modest and heavy loading cases, Figs. 8(a)-8(b), the asymptotic theories are superior to the KaneMindlin theory. Since this relation holds even for the $h$ theory, this is an unexpected outcome. However, for even higher frequencies, the results due to Kane-Mindlin are better than the asymptotic $h$ theory. The different approximate equations behave quite poorly 
in the steel-air case, Fig. $8(\mathrm{c})$, especially close to $x=0$. This is probably due to the unphysical behavior of these approximate equations near a symmetric point load for a free plate, rendering infinite displacements.

\section{CONCLUSIONS}

In this paper the derivation of approximate boundary conditions for an elastic layer immersed in a fluid is presented. Starting from the three-dimensional equations of motion, the boundary conditions are stated in terms of the plate fields together with the pressure field and its normal derivative. By expressing the sums and differences of the boundary conditions, the terms involving the plate fields are expanded in Maclaurin's series in the plate thickness. In order to eliminate the plate fields (and their normal derivatives), the threedimensional equations of motion (and normal derivatives thereof) are adopted. This gives two different linear systems; one antisymmetric involving $\Delta p$ and $\Sigma \partial_{z} p$ and one symmetric involving $\Sigma p$ and $\Delta \partial_{z} p$. The operators relating $\Delta p$ to $\Sigma \partial_{z} p$ and $\Sigma p$ to $\Delta \partial_{z} p$, respectively, are expressed in terms of time derivatives, spatial derivatives in the layer plane and power series in the plate thickness. These equations can be truncated to any desired order, and the explicit relations up to and including order five are presented. Comparisons are made with different classic plate theories. Especially, the often used antisymmetric equations due to Kirchhoff and Mindlin are here of interest.

In the numerical results, both the reflection and transmission from plane waves as well as the boundary pressure fields from line forces are studied. The results using exact 
three-dimensional theory, classical flexural equations (e.g. Kirchhoff, Mindlin) as well as asymptotic expansions $\left(h, h^{3}, h^{5}\right)$ are compared. Three different cases are studied: modest loading (aluminum-water), heavy loading (polyethylene-water) and light loading (steelair). In the planar scattering problems, the classical flexural theories are rather good for modest and light loading as the antisymmetric motion is prevailing. However, in the heavy loading example, the importance of including compressional motion is obvious. Taking all the three loading cases into consideration, the asymptotic $h^{3}$ and $h^{5}$ expansions theories give more satisfying results than the different classic theories. In the line loading cases, purely antisymmetric and symmetric motions are examined separately. The accuracies of the Kirchhoff theory in the antisymmetric case is inferior to the other theories of order three (Mindlin and asymptotic $h^{3}$ ), which in turn are inferior to the asymptotic $h^{5}$ solutions. In the symmetric case, all the displayed results are here of the same order.

\section{ACKNOWLEDGMENTS}

The present work is partially supported by the Swedish Research Council (VR) and the Volvo-Chalmers Vehicle Research Program.

\section{References}

[1] P. Bövik, "On the modelling of thin interface layers in elastic and acoustic scattering problems," Q. J. Mech. Appl. Math. 47(1), 17-42 (1994). 
[2] H. Ammari, and S. He, "Generalized effective impedance boundary conditions for an inhomogeneous thin layer in electromagnetic scattering," J. Electromagn. Waves Appl. 11, 1197-1212 (1997).

[3] P. Olsson, S. K. Datta, and A. Boström, "Elastodynamic scattering from inclusions surrounded by thin interface layers," J. Appl. Mech. 57, 672-676 (1990).

[4] S. I. Rokhlin and W. Huang, "Ultrasonic wave interaction with a thin anisotropic layer between two anisotropic solids. II Second-order asymptotic boundary conditions," J. Acoust. Soc. Am. 94(6), 3405-3420 (1993).

[5] G. Johansson and A. J. Niklasson, "Approximate dynamic boundary conditions for a thin piezoelectric layer," Int. J. Solids Structures 40(13-14), 3477-3492 (2003).

[6] P. M. Morse and K. U. Ingard, Theoretical acoustics (McGraw-Hill Book Company, New York, 1968).

[7] R. D. Mindlin, "Influence of rotatory inertia and shear on flexural motion of isotropic, elastic plates," J. Appl. Mech. 18, 31-38 (1951).

[8] B. L. Woolley, "Acoustic radiation from fluid-loaded elastic plates I. Antisymmetric modes," J. Acoust. Soc Am. 70(3), 771-781 (1981).

[9] B. L. Woolley, "Acoustic radiation from fluid-loaded elastic plates II. Symmetric modes," J. Acoust. Soc Am. 72(3), 859-869 (1982). 
[10] G. Jemielita, "On kinematical assumptions of refined theories of plates: A survey," J. Appl. Mech. 57, 1088-1091 (1990).

[11] A. L. Goldenveizer, J. D. Kaplunov, and E. V. Nolde, "On Timoshenko-Reissner type theories of plates and shells," Int. J. Solids Structures 30(5), 675-694 (1993).

[12] N. A. Losin, "Asymptotics of flexural waves in isotropic elastic plates," Transactions of the ASME 64, 336-342 (1997).

[13] N. A. Losin, "Asymptotics of extensional waves in isotropic elastic plates," Transactions of the ASME 65, 1042-1047 (1998).

[14] A. Boström, G. Johansson, and P. Olsson, "On the rational derivation of a hierarchy of dynamic equations for a homogeneous, isotropic, elastic plate," Int. J. Solids Structures 38(15), 2487-2501 (2001).

[15] A. Freedman, "Reflectivity and transmittivity of elastic plates, I: Comparison of exact and approximate theories," J. Sound Vib. 59(3), 369-393 (1978).

[16] R. Fiorito, W. Madigosky, and H. Überall, "Resonance theory of acoustic waves interacting with an elastic plate," J. Acoust. Soc. Am. 66(6), 1857-1866 (1979).

[17] P. S. Dubbelday and A. J. Rudgers, "An analysis of effective shear modulus for flexural and extensional waves and its application to reflection of sound by a plate," J. Acoust. Soc. Am. 70(2), 603-614 (1981). 
[18] D. E. Chimenti and S. I. Rokhlin, "Relationship between leaky Lamb modes and reflection coefficient zeroes for a fluid-coupled elastic layer," J. Acoust. Soc. Am. 88(3), 1603-1611 (1990).

[19] A. D. Stuart, "Acoustic radiation from submerged plates. I. Influence of leaky wave poles," J. Acoust. Soc. Am. 59(5), 1160-1169 (1976).

[20] Y. D. Sergeev, "Influence of shear, rotary inertia, and longitudinal vibrations on the radiation of sound by a point-driven plate," Sov. Phys. Acoust. 25(2), 174-176 (1979).

[21] A. J. Rudgers, P. S. Dubbelday, and L. A. Fagerstrom, "Extensional-wave and flexuralwave contributions to the sound field radiated by a fluid-loaded infinite plate," J. Acoust. Soc. Am. 80(3), 932-950 (1986).

[22] A. J. Langley, "Exact and approximate theories for fluid-loaded, infinite, elastic plates," J. Acoust. Soc. Am. 83(4), 1366-1376 (1988).

[23] A. J. DiPerna and D. Feit, "An approximate Green's function for a locally excited fluid-loaded thin elastic plate," J. Acoust. Soc. Am. 114(1), 194-199 (2003).

[24] T. R. Kane and R. D. Mindlin, "High-frequency extensional vibrations of plates," J. Appl. Mech. 23, 277-283 (1956).

[25] L. M. Lyamshev, "Reflection of sound from a moving thin plate," Sov. Phys. Acoust. 6(4), 505-507 (1960). 
[26] E. L. Shenderov, "Sound transmission through a transversely isotropic plate," Sov. Phys. Acoust. 30(1), 69-73 (1984).

[27] J. D. Achenbach, Wave Propagation in Elastic Solids (North-Holland, Amsterdam, 1973).

[28] N. A. Losin, "On the equivalence of dispersion relations resulting from RayleighLamb frequency equation and the operator plate model," Transactions of the ASME 65, 1042-1047 (1998). 


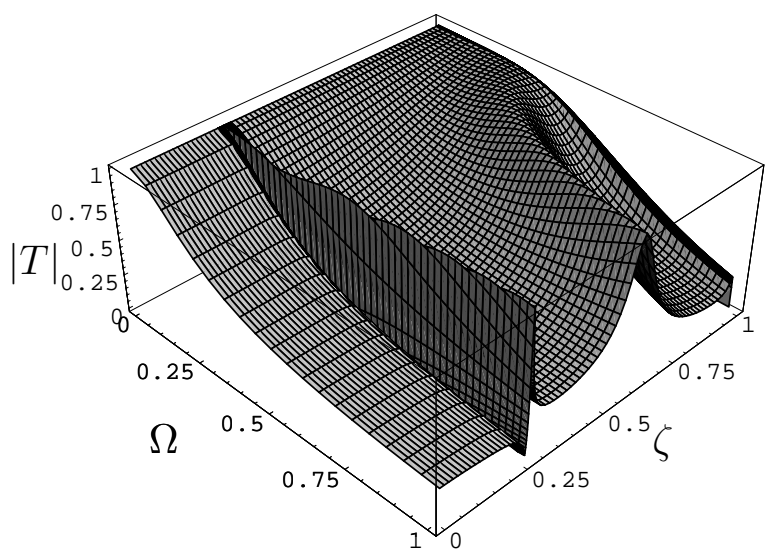

(a) Modulus of $T$.

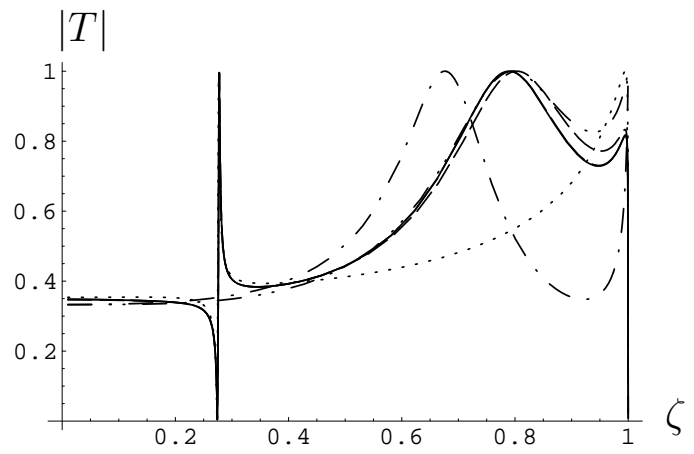

(c) $\Omega=0.5$

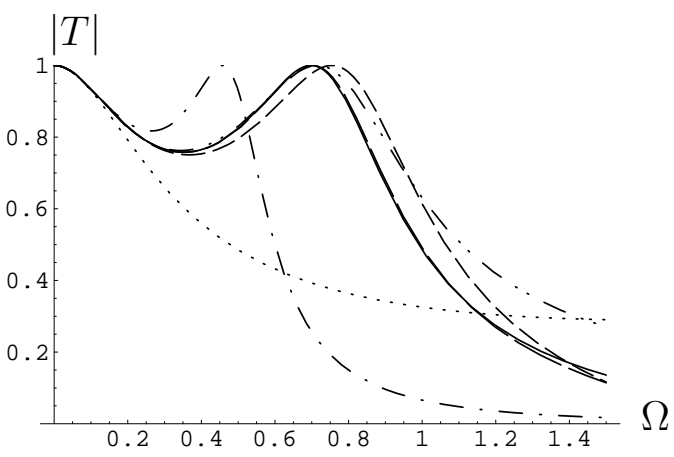

(b) $\phi=45^{\circ}$.

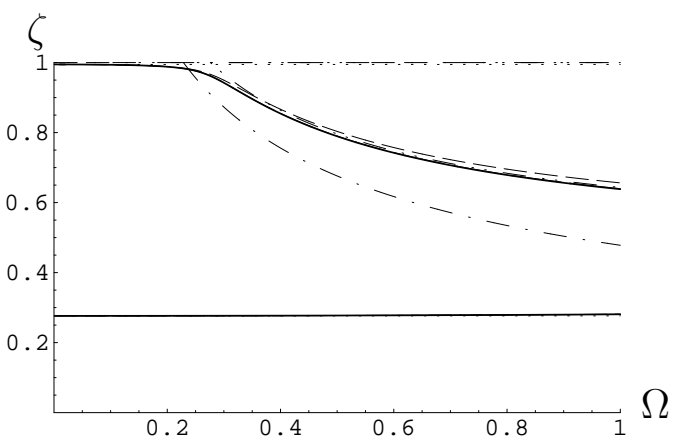

(d) $|T|=1$

Figure 1: Aluminum-Water. —— exact, $--h^{5},---h^{3}, \cdots h,-\cdot-$ Kirchhoff, $-\cdots-$ Mindlin. 


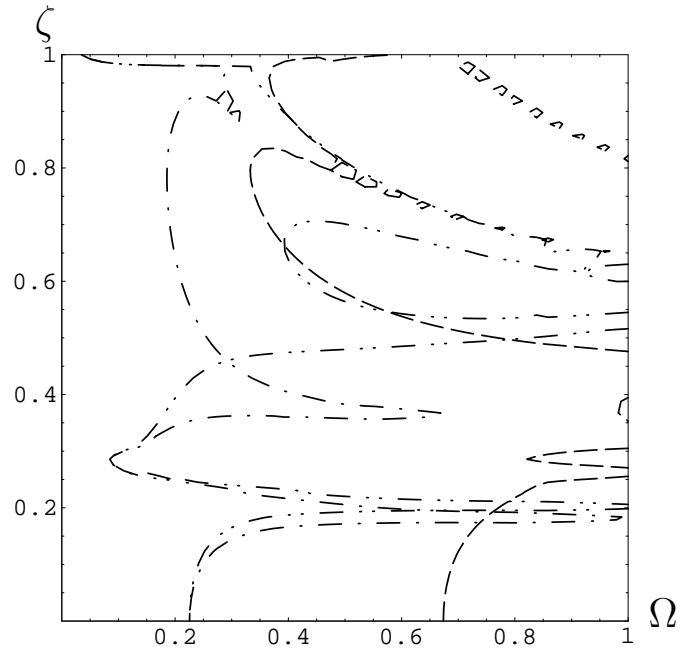

(a) $1 \%$ relative error contours for $|T|$.

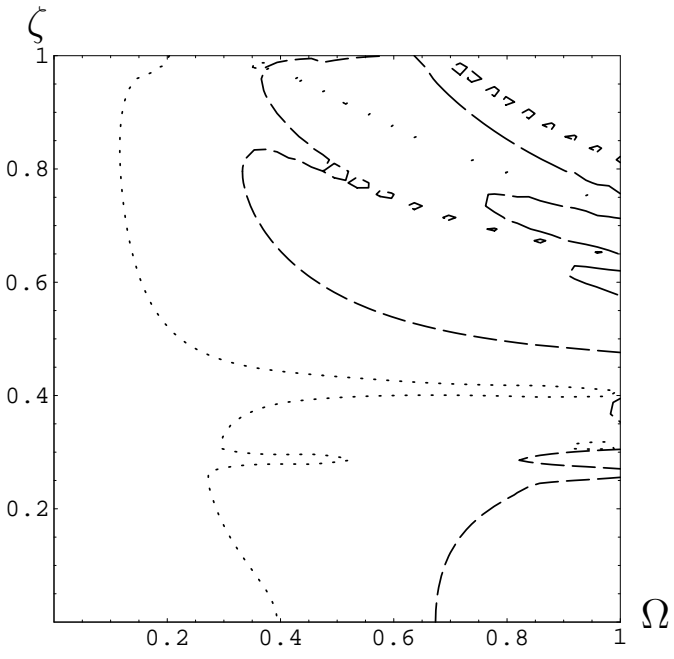

(b) $1 \%$ relative error contours for $|T|$.

Figure 2: Aluminum-Water. $--h^{5},---h^{3}, \cdots h,-\cdot-$ Kirchhoff, $-\cdots-$ Mindlin. 


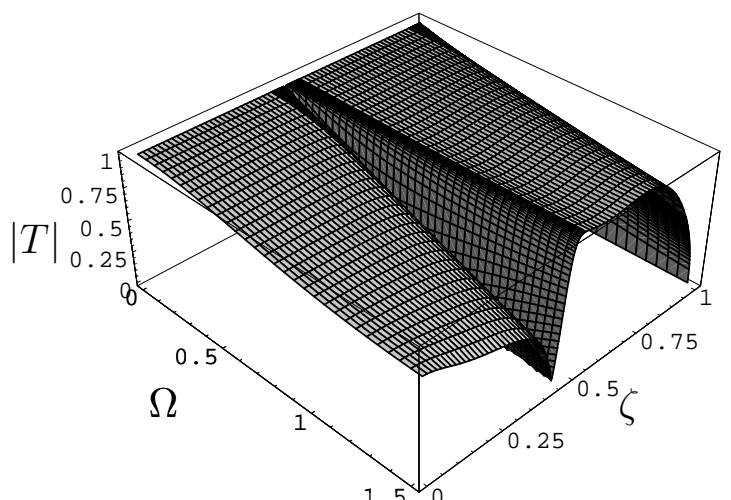

(a) Modulus of $\mathrm{T}$

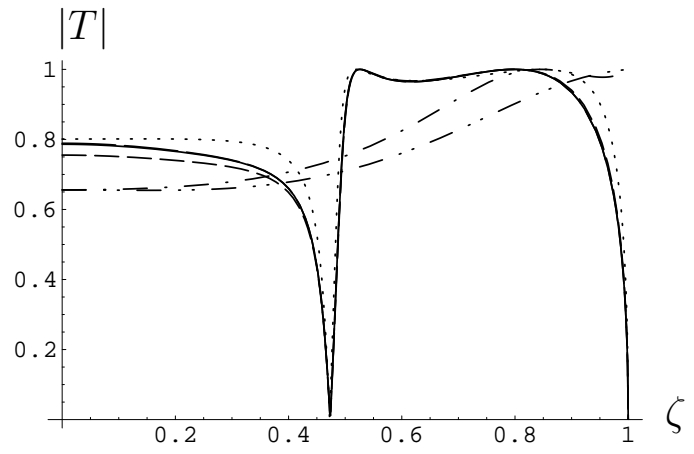

(c) $\Omega=1$

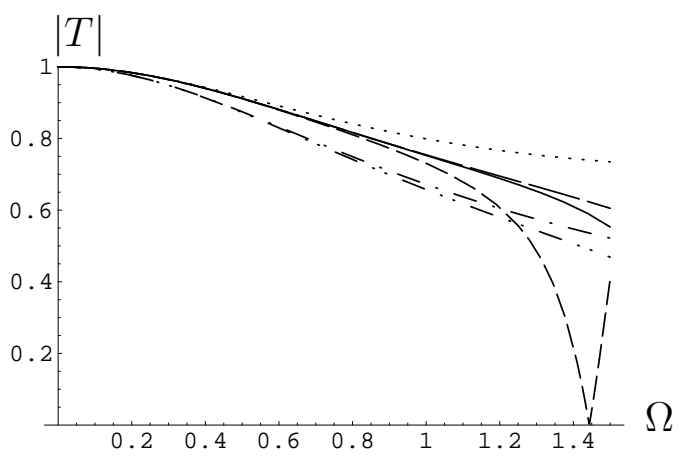

(b) $\phi=15^{\circ}$

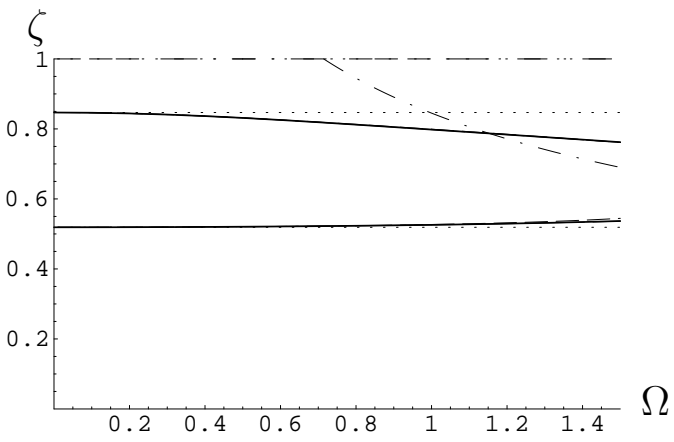

(d) $|T|=1$

Figure 3: Polyethylene-Water. exact, $h^{5},---h^{3}, \cdots h,-\cdot-$ Kirchhoff, - . - Mindlin. 

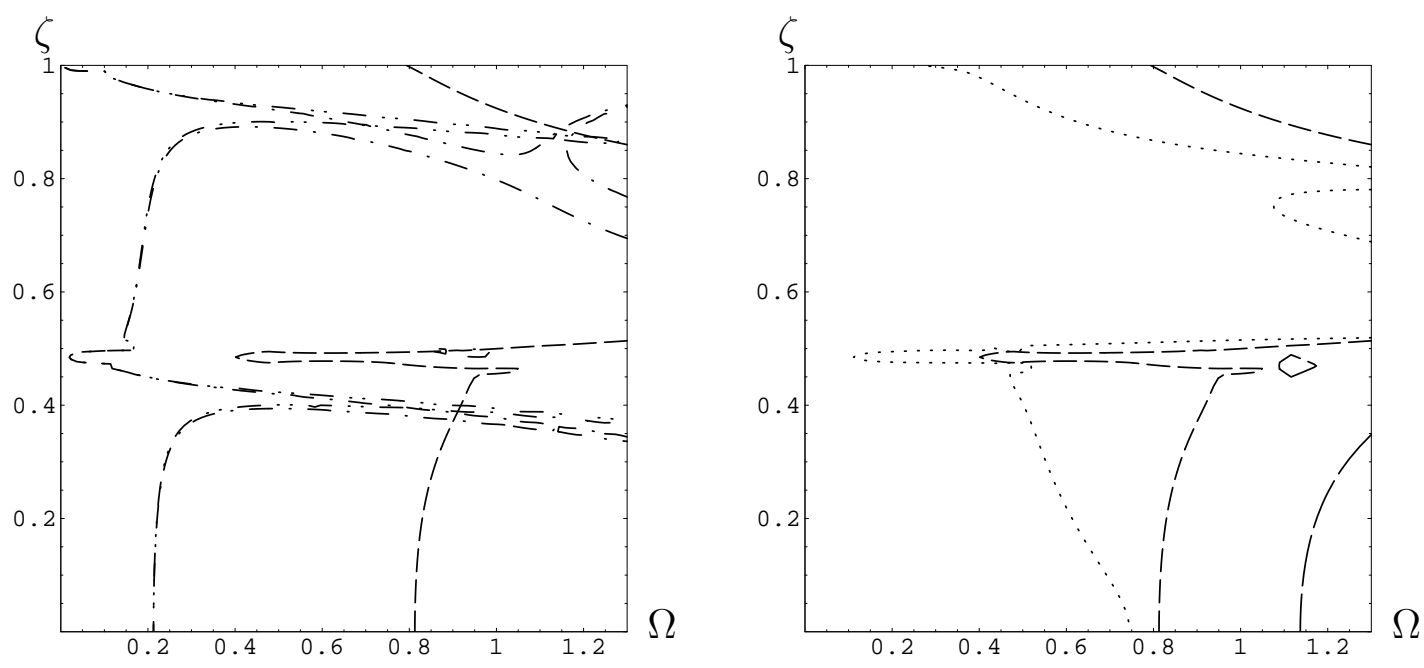

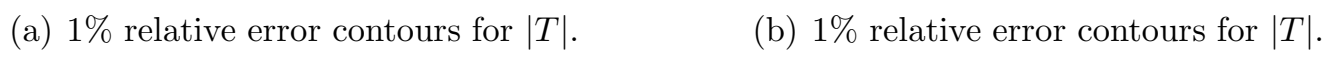

Figure 4: Polyethylene-Water. - - $h^{5},---h^{3}, \cdots h,-\cdot-$ Kirchhoff, $-\cdots-$ Mindlin.
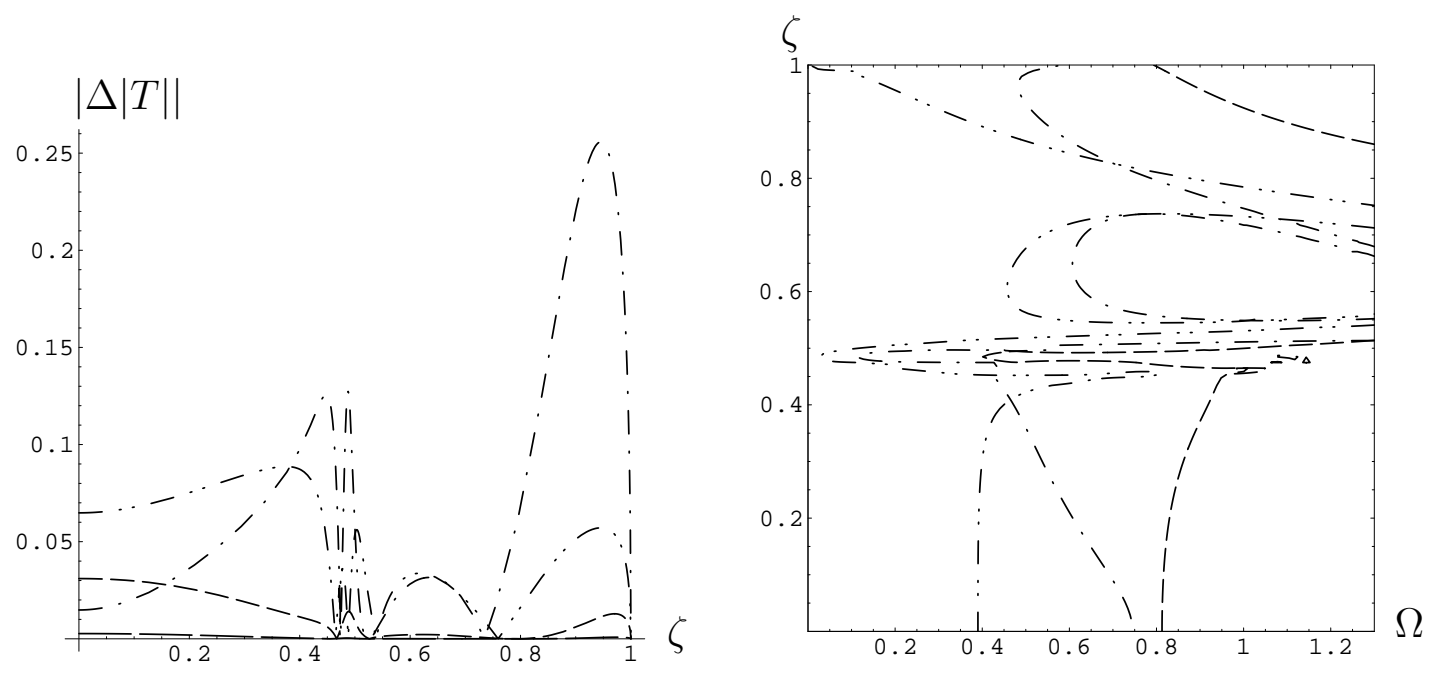
(a) $\Omega=1$
(b) $1 \%$ relative error contours for $|T|$.

Figure 5: Polyethylene-Water. - - $h^{5},---h^{3},-\cdot-\mathrm{K}$-GPS, $-\cdots-\mathrm{M}-\mathrm{KM}$. 

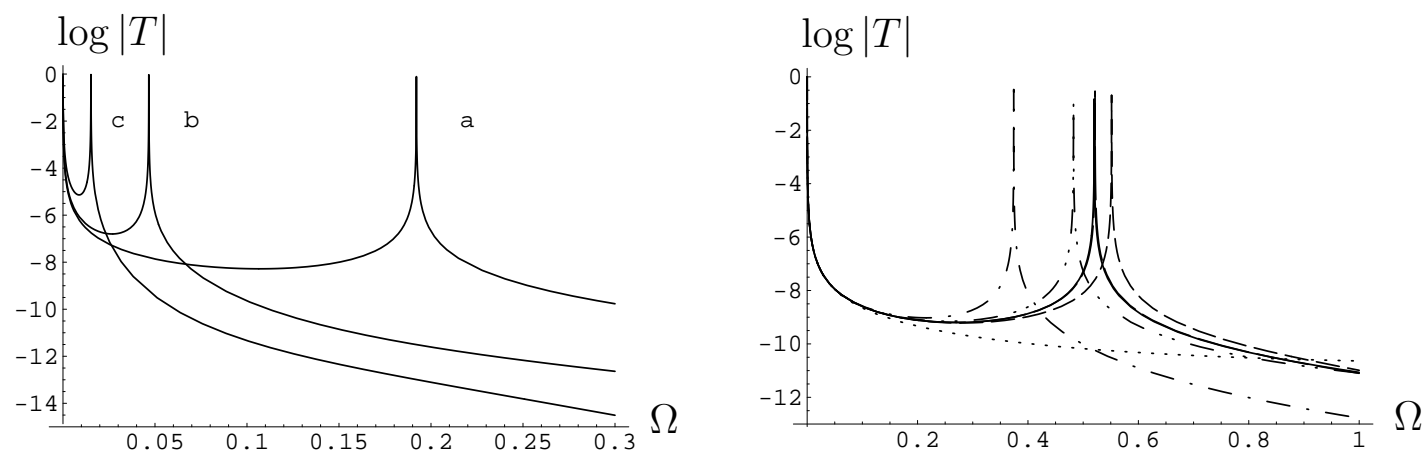

(a) Modulus of $\mathrm{T}$ for a: $\phi=15^{\circ}, \mathrm{b}: \phi=30^{\circ}$ and

(b) $\phi=10^{\circ}$

c: $\phi=60^{\circ}$
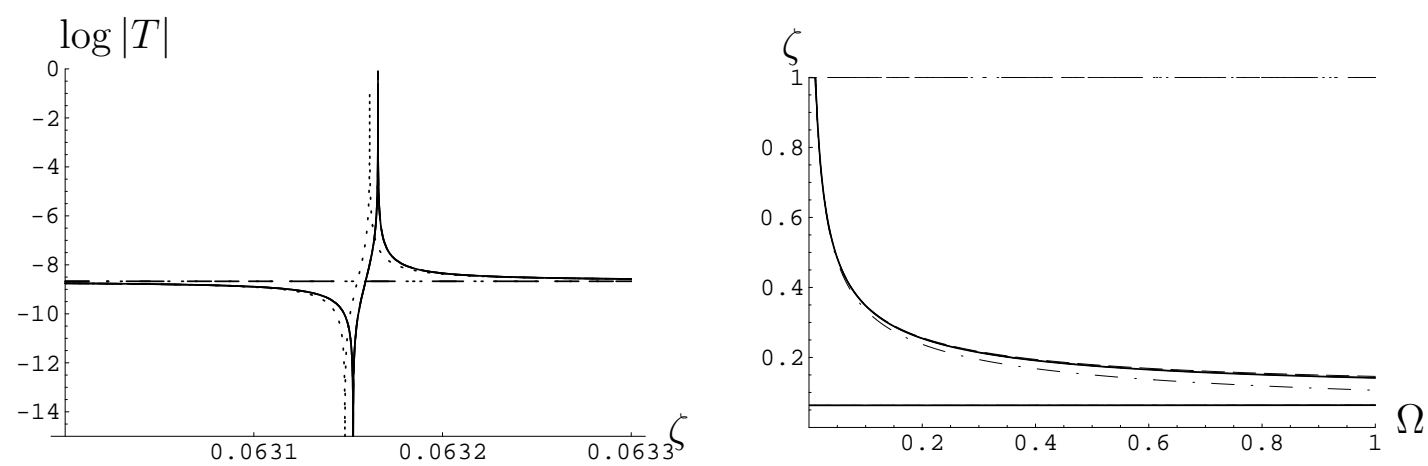

(c) $\Omega=0.1$

(d) $|T|=1$

Figure 6: Steel-Air. — exact, $--h^{5},---h^{3}, \cdots h,-\cdot-$ Kirchhoff, $-\cdots-$ Mindlin. 


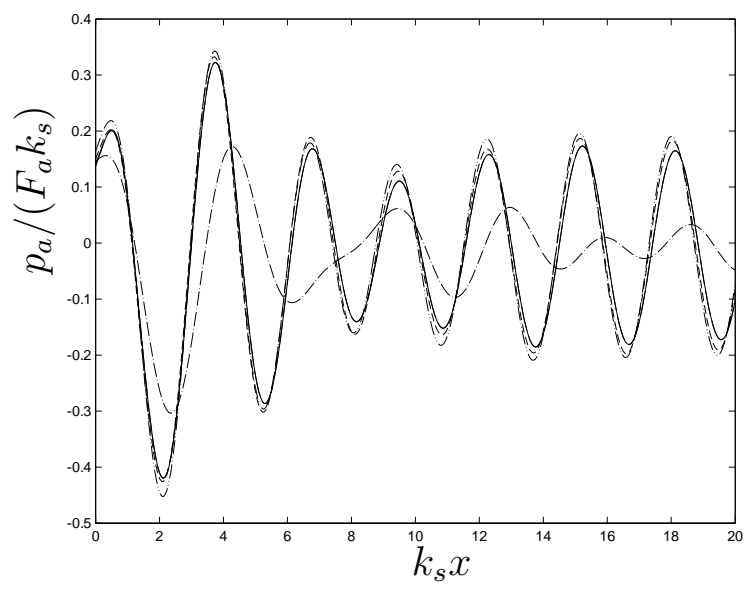

(a) Aluminum-Water.

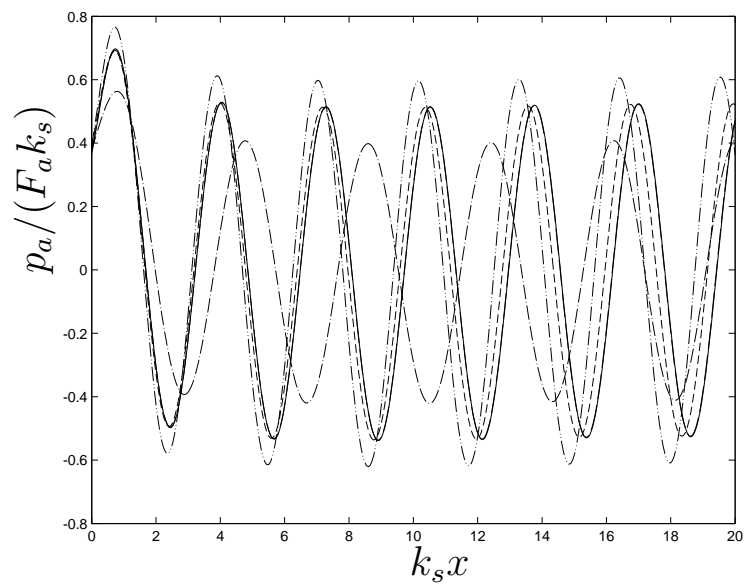

(b) Polyethylene-Water.

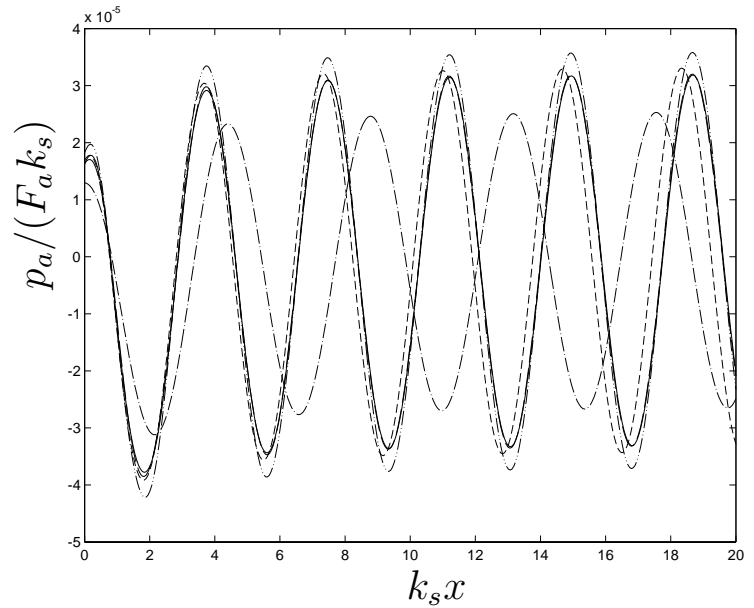

(c) Steel-Air.

Figure 7: Antisymmetric plate boundary press 3 sure $p_{a} /\left(F_{a} k_{s}\right)$ as a function of distance $k_{s} x$ for $k_{s} h=1$. - exact, - - $h^{5},---h^{3},-\cdot-$ Kirchhoff, $-\cdots-$ Mindlin. 


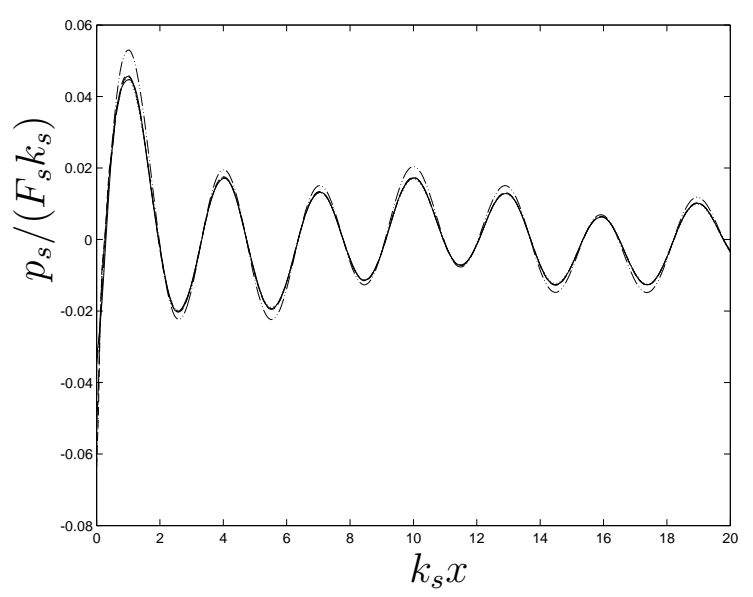

(a) Aluminum-Water.

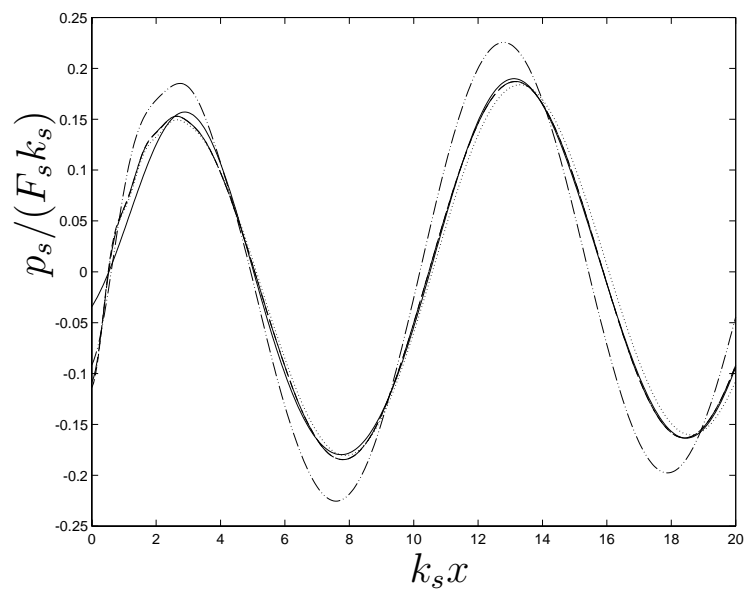

(b) Polyethylene-Water.

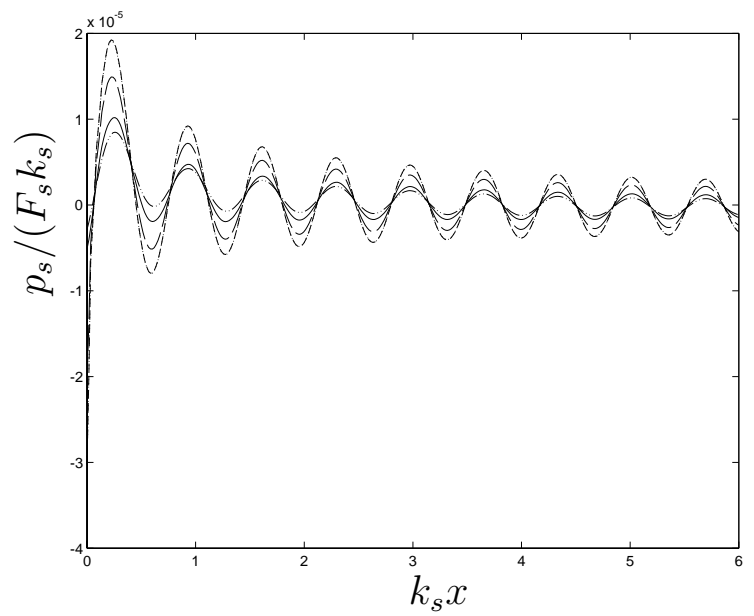

(c) Steel-Air.

Figure 8: Symmetric plate boundary pressure $p_{s} /\left(F_{s} k_{s}\right)$ as a function of distance $k_{s} x$ for $k_{s} h=1 .-$ exact, $--h^{5},---h^{3}, \cdots h,-\cdots-$ Kane-Mindlin. 